\title{
ENERGY EFFICIENCY IN WIRELESS SENSOR NETWORKS USING MOBILE BASE STATION
}

\author{
Dorottya Vass*, Zoltán Vincze, Rolland Vida and Attila Vidács ${ }^{\dagger}$ \\ Dept. of Telecommunications and Media Informatics \\ Budapest University of Technology and Economics \\ Magyar tudósok krt. 2, 1117 Budapest, Hungary \\ $\{$ vass,vinczez,vida,vidacs\}@tmit.bme.hu
}

\begin{abstract}
A sensor network consists of a large number of small, low-cost devices with sensing, processing and transmitting capabilities. The sensor nodes have limited battery power; therefore energy efficiency is a critical design issue. In this paper we propose to move the sink node, called Base Station (BS) so as to decrease the energy consumption of the whole network. We present two possible strategies to move the BS: the first one minimizes the average consumed energy, while the other one minimizes the maximum transmission energy for every active sensor. To evaluate the performance of the two strategies, we compare these with the case, when the BS is deployed in a fixed position. Simulation results show that the proposed processes can reduce energy consumption, thereby significantly extending the lifetime of the entire sensor network.
\end{abstract}

Keywords: sensor network, mobility, mobile base station, energy efficiency

\section{Introduction}

A sensor network consists of a large number of small, low-cost devices with sensing, processing, and transmitting capabilities. The main goal of the operation is to observe a region and gather and relay information to a sink node or set of sink nodes, called Base Station (BS). Forwarding the data to the BS is possible in two ways: using direct or multihop communication. In the first case every sensor transmits its data directly to the sink; in the second case, the sensors are communicating with the neighbors that forward the information in the direction of the sink.

\footnotetext{
*This work has been partly supported by the European Union under the E-Next Project FP6-506869 and by the Inter-University Centre for Telecommunications and Informatics.

${ }^{\dagger}$ A. Vidacs is with the Research Group for Informatics and Electronics of the Hungarian Academy of Sciences, grantee of the János Bolyai Scholarship.
} 
The sensor networks can be also categorized by the periodicity of data transmissions. In a time-driven network every node sends messages periodically, while in an event-driven one a node sends message only when sensing a phenomenon [Yao and Gehrke, 2002]. The third category is the query-driven approach, when the sensors send data only after receiving a query from the BS. There are also hybrid networks that combines the previous three models.

The sensors are usually deployed densely and often on-the-fly [Gandham et al., 2004]. They operate untethered and unattended, are limited in power, computational capacities and memory. Because of these constraints the sensor network must have efficient self-organizing capabilities, while optimising energy consumption.

A primary design issue in sensor networks is energy efficiency. The sensors are small-sized, cheap, and usually deployed in inaccessible regions; therefore they are supplied only by a small battery which is impossible (or very costly) to recharge. The main goal is to prolong the lifetime of the network, which can be defined in several ways:

- the time when the first node depletes its battery,

- the time till a given percentage of the sensors has enough energy to operate,

- the time till a given percentage of the region is covered by alive sensors.

Communicating 1 bit over a wireless medium at short ranges consumes far more energy than processing that bit. With the current technology, the energy consumption for communication is several magnitude higher, than the energy required for computation; and wireless communication is foreseen to continue to dominate energy consumption in the near future [Doherty et al., 2001]. There are two possible ways to decrease the energy used for communication in a sensor network: minimize the amount of the transmitted data, or shorten the communication range. The transmission energy is proportional to $d^{\alpha}$, where $d$ is the transmission distance, while $\alpha$ is the attenuation exponent. Due to multipath and other interference effects, $\alpha$ is typically in the range of 2 to 5 [Zhao and Gubias, 2004]. Thus, minimizing the amount and the range of communication as much as possible can significantly prolong the life of a sensor network.

In order to decrease the transmission distance in sensor networks, we propose an approach where the BS is capable to change its position. The BS tries to find the optimal location as far as energy consumption concerned, using two optimization strategies: the first one minimizes the average consumed energy, while the other one minimizes the maximum transmission energy for every active sensor. We evaluated the strategies using simulation, the results showing, that they significantly prolong the lifetime of the network. 
The rest of the paper is organized as follows: Section 2 summarizes the related work. Section 3 introduces the concept of the mobile BS and the two strategies to locate the optimal location of the BS. The results of the simulations evaluating the performance of the strategies are presented in Section 4. Finally, Section 5 sketches the conclusions and presents some tasks for the future work.

\section{Related Work}

Recently, energy efficiency has been one of the focus points of research in the field of sensor networks. There have been a lot of proposals to minimize energy consumption in sensor networks [Intanagonwiwat et al., 2000] [Gan et al., 2004] [Ganesan et al., 2001] [Schurgers and Srivastava, 2001] [Rahul and Rabaey, 2002]. These proposals are common in the fact that they all assume the BS and the sensor nodes to be static.

The majority of the research papers that appeared until now consider sensor networks to be entirely static. However, some consider that these networks should be able to cope with the mobility of either the BS or the sensor node themselves [Al-Karaki and Kamal, 2004]. This new approach raises the possibility of developing new methods to spare energy.

There are some papers which consider only the sensors to be mobile. On the one hand, Howard et al. present an incremental deployment algorithm for mobile sensor networks [Howard et al., 2002]. On the other hand, Rahimi et al. study the feasibility of extending the lifetime of a wireless sensor network by exploiting mobility [Rahimi et al., 2003]. They assume that a small percentage of network nodes are autonomously mobile, allowing them to move in search of energy, recharge, and deliver energy to immobile, energy-depleted nodes.

The idea of mobile BS is also considered by some papers. Ye et al. propose to build an overlay network in the sensor network for data dissemination to mobile sinks [Ye et al., 2002]. Kim et al. are examining the same problem, they suggest a technique to build dissemination tree for disseminating data to mobile sinks [Kim et al., 2003]. The mobility of the BS is unpredictable in these works (e.g. sinks move according to the random waypoint model).

Other papers are dealing with the task of moving the BS in the sensor network in order to elongate the lifetime of the network. Wang et al. suggest a linear programming method to determine the optimal movement of the BS and the sojourn time at different points in the network to maximize the network lifetime [Wang et al., 2005]. The authors assume the sensors are deployed in the crossing points of a bi-dimensional square grid and the BS can move only on the grid from one node to another. Gandham et al. propose to decrease energy consumption, thus increasing the network lifetime using multiple mobile Base Stations [Rao et al., 2003]. The number of the base stations is known a priori 
and they can be located only at a priori given sites on the border of the sensor network. The goal is to find the optimal place of the base stations regarding to energy consumption in order to prolong network lifetime. The common in the previous works is that the authors consider only proactive (time-driven) sensor networks where each node generates equal amount of data per time unit.

In our work we also consider the mobility of the base station. However, as opposed to the previous assumptions, we consider the BS can move anywhere inside the sensor network (for example the BS is mounted on a robot plane). The type of the network we considered is also different, hence we assumed event-driven network where the sensor sends data only when sensing an event.

\section{Strategies for Moving Mobile Base Station}

In this work, we consider a typical sensor network, where the nodes have no mobility after the deployment, and operate in an event-driven way. The network is divided into small clusters, in which we assume the sensors are distributed uniformly. Each cluster has a clusterhead, this is the BS. The small size of the clusters makes it possible for sensors in the cluster to communicate with the BS directly. We assume that every sensor knows its distance from the BS.

Although sensing also requires energy, this is far less, than the energy used for communication. Therefore in this paper we deal only with the transmission energy. The energy used for the communication is proportional to $d^{\alpha}$, where $d$ is the transmission distance and $\alpha$ is the attenuation parameter. Sensors are able to change the level of the transmission energy depending on their distance $d$.

We consider that the BS is mobile and is able to reach every point in the area of the cluster. It has relatively sufficient energy, because it has access to power supply. We assume that the BS moves fast enough to reach the optimal location very rapidly (practically 'immediately' after calculating the location).

In this paper we examine two strategies for moving the BS, and compare their performance with the case when the BS is fixed.

\subsection{Minimizing the average energy consumption}

This strategy minimizes the energy consumed by the active sensors in the network at every moment once an event happens. Let $\left(x_{0}, y_{0}\right)$ denote the coordinates of the BS, and $\left(x_{i}, y_{i}\right)$ the coordinates of the $i$ th $(i=1 \ldots n)$ sensor. The distance $d_{i}$ between the BS and the $i$ th sensor is

$$
d_{i}=\sqrt{\left(x_{0}-x_{i}\right)^{2}+\left(y_{0}-y_{i}\right)^{2}} .
$$


Let $E_{t i}$ be the energy used by the $i$ th sensor for the communication with the BS and let $E_{0}$ be the energy needed to transmit one unit of data. Then if the $i$ th sensor transmits one unit of data

$$
E_{t_{i}}=E_{0} d_{i}^{\alpha}, \quad \alpha \in[2,5] .
$$

If $V$ is the set of sensors, and $A \subset V$ is the set of active nodes, then the energy consumed by the active sensors is

$$
E_{t}=E_{0} \sum_{i \in A} d_{i}^{\alpha}
$$

The method places the BS where this sum reaches its minimum:

$$
\left(x_{0}, y_{0}\right)=\arg \min _{x, y} E_{t} .
$$

In the rest of the paper we will refer to this strategy as 'minavg'. The idea here is that using this strategy causes the network to always spend the minimal energy for the communication between the active sensors and the BS. Therefore the total energy of the network will remain the highest compared to other strategies.

The used total energy is minimal when

$$
\left.\frac{\partial E}{\partial x}\right|_{x=x_{0}}=0 \text { and }\left.\frac{\partial E}{\partial y}\right|_{y=y_{0}}=0 .
$$

The partial derivatives are

$$
\begin{aligned}
\frac{\partial E}{\partial x} & =\frac{\partial}{\partial x}\left(E_{0} \sum_{i \in A} d^{\alpha}\right) \\
& =E_{0} \sum_{i \in A} \frac{\partial}{\partial x}\left(\sqrt{\left(x_{0}-x_{i}\right)^{2}+\left(y_{0}-y_{i}\right)^{2}}\right) \\
& =E_{0} \sum_{i \in A} \alpha\left[\left(x_{0}-x_{i}\right)^{2}+\left(y_{0}-y_{i}\right)^{2}\right]^{\frac{\alpha-1}{2}} \\
& \times \frac{\partial}{\partial x}\left(\sqrt{\left(x_{0}-x_{i}\right)^{2}+\left(y_{0}-y_{i}\right)^{2}}\right) \\
& =E_{0} \alpha \sum_{i \in A}\left(x_{0}-x_{i}\right)\left[\left(x_{0}-x_{i}\right)^{2}+\left(y_{0}-y_{i}\right)^{2}\right]^{\frac{\alpha-2}{2}}
\end{aligned}
$$

and similarly,

$$
\frac{\partial E}{\partial y}=E_{0} \alpha \sum_{i \in A}\left(y_{0}-y_{i}\right)\left[\left(x_{0}-x_{i}\right)^{2}+\left(y_{0}-y_{i}\right)^{2}\right]^{\frac{\alpha-2}{2}} .
$$

Unfortunately there is no closed formula solution to find the optimal $\left(x_{0}, y_{0}\right)$ coordinates, thus it has to be determined using optimisation methods (for example some kind of gradient-based search [Carson and Maria, 1997]). 


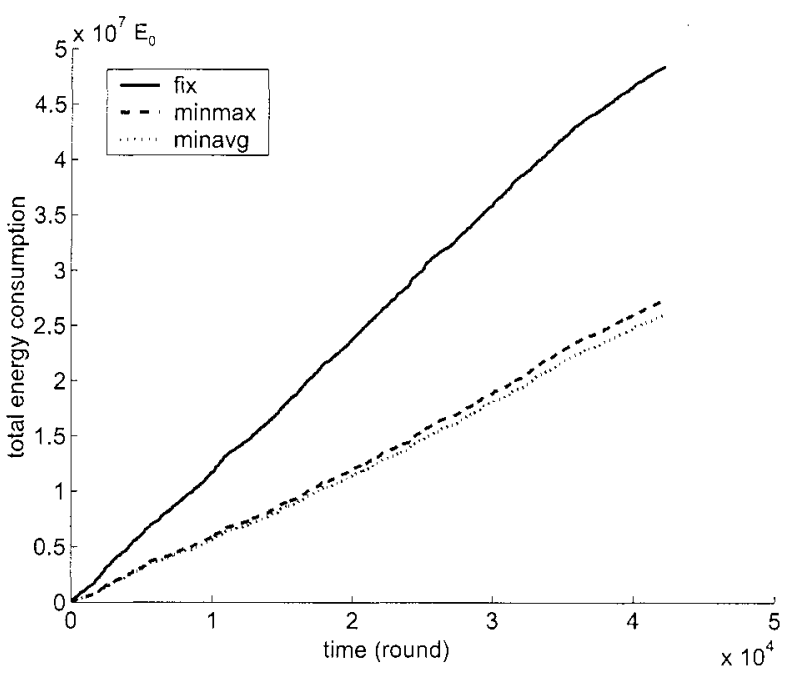

(a) $p=0.0005$

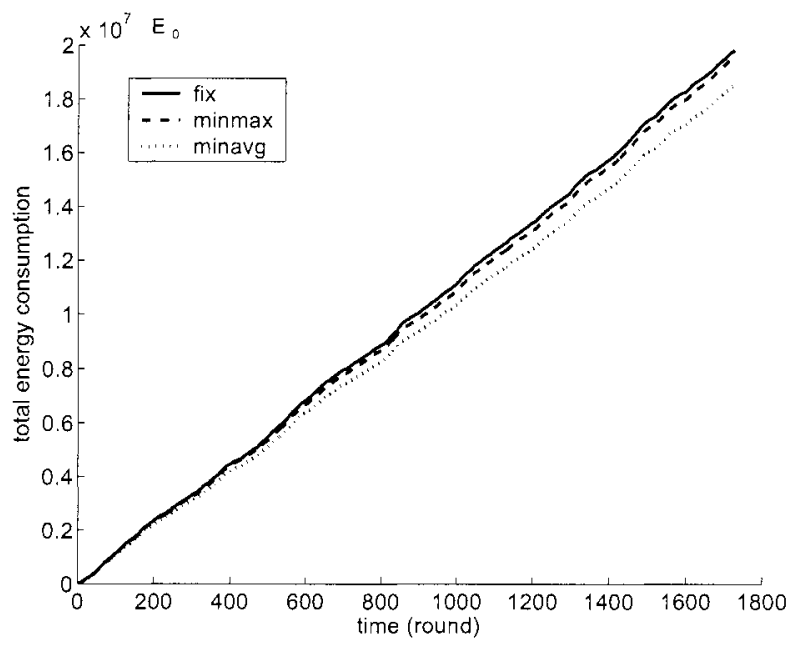

(b) $p=0.005$

Figure 1. The total energy consumption of the network 


\subsection{Minimizing the maximum energy consumed by an active sensor}

The drawback of the minavg approach is that it does not take into account the interests of the individual sensors. For example, it may happen that most of the active sensors are close to the BS, while one or more nodes are far from it. Therefore these sensors use much more energy than the others and deplete their battery sooner, so they have less lifetime.

The strategy introduced in this subsection is able to avoid this problem. In order to do so, it minimizes the maximum transmission energy for the sensors in the network; i.e.,

$$
\max _{i \in A} E_{t_{i}} \rightarrow \min
$$

hence, energy consumption will be more balanced.

As the transmission energy depends on the distance between the sensor and the BS, this strategy is equivalent with minimizing the maximum distance between the BS and every active sensor in the network. The location of the BS is given by the coordinates minimizing the next expression:

$$
\left(x_{0}, y_{0}\right)=\arg \min _{x, y}\left[\max _{i \in A} \sqrt{\left(x_{0}-x_{i}\right)^{2}+\left(y_{0}-y_{i}\right)^{2}}\right] .
$$

In the rest of the paper we will refer to this strategy as 'minmax'.

The optimisation task is equivalent to the problem of finding the minimal enclosing circle, where we have a set of points and the task is to find the minimum radius circle that encloses all of them. There are several algorithms to solve this problem. The simplest one considers every circle being defined by two or three of the $n$ points, and finds the smallest of these circles that contains every point. This solution has a total running time of $O\left(n^{4}\right)$. On the other hand it has been shown that the minimal enclosing circle problem can be solved in $O(n)$ time using the prune-and-search techniques for linear programming [Megiddo, 1983].

\section{Simulation Results}

In the simulations we considered the sensor network introduced in Section 3. Recall that we assumed a network divided into small clusters, every cluster having a BS. Inside the cluster we assumed that the sensors are distributed uniformly; therefore, we used a 20 size grid topology for the simulations, where the sensors were situated in the points of the grid. During the simulations we examined the performance of the BS placement strategies in one cluster.

Recall that the transmission energy is $E_{t} \sim \sum d_{i}^{\alpha}$, where we chose $\alpha$ to be 3 in the simulations. The time was split into equal periods and we assumed that 


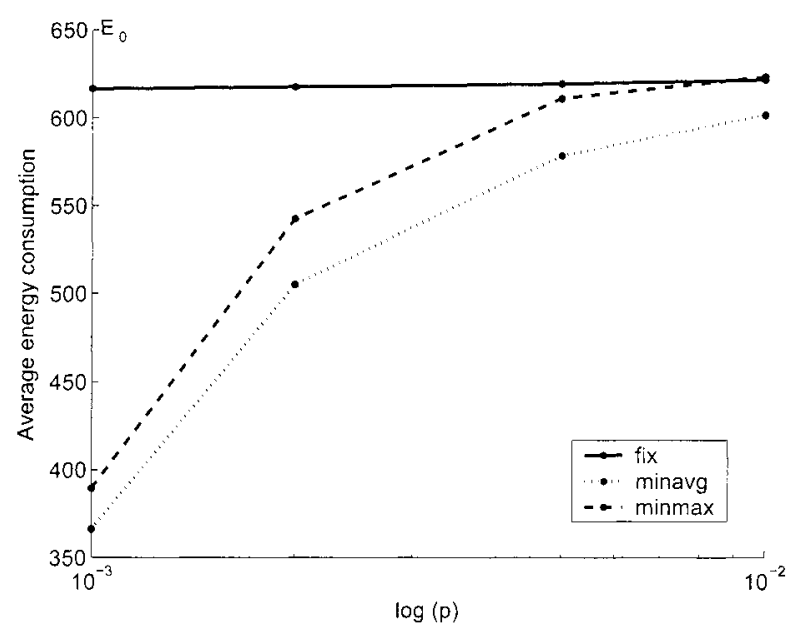

Figure 2. The average energy consumption of one active sensor in one round

an event can occur or end only at the beginning of the time period. Every active sensor sends the same amount of data in a round. The BS can be relocated also at the start of a round.

We simulated an event-driven network, hence a sensor sends data only when sensing an event. We considered that an event can occur in a uniformly distributed manner in the area of the cluster and it is sensed only by one sensor. During a round, an event appeared in the region of a sensor with probability $p$. The duration of event is geometrically distributed, i.e., an existing event persisted in the next round with probability $q$. Therefore, every sensor became active independently with probability $p$; after becoming active, the sensor stayed in active state in every following round with the probability $q$. In the simulations $p$ was in the range of 0.0005 to 0.01 , while $q$ was 0.9 . During the simulations the initial energy of every sensor was $300 \mathrm{~kJ}$, the distance of the points of the grid was $5 \mathrm{~m}$, every active sensor sent one unit of data in every round, and $E_{0}$ was $0.25 \mathrm{~mJ}$.

For the simulation we used the MATLAB environment. To evaluate the performance of the two mobile BS strategies, we compare these with the case when the BS is deployed in a fix position. Since in our considerations every node becomes active with the same probability, the best place for the fixed BS is the center of the network.

Figure 1 presents the total energy consumption of the whole network, in the case when sensors became active with the probability of $p=0.0005$ and $p=0.005$, respectively. In both cases $\mathrm{q}$ is 0.9 . In the first case two sensors are 


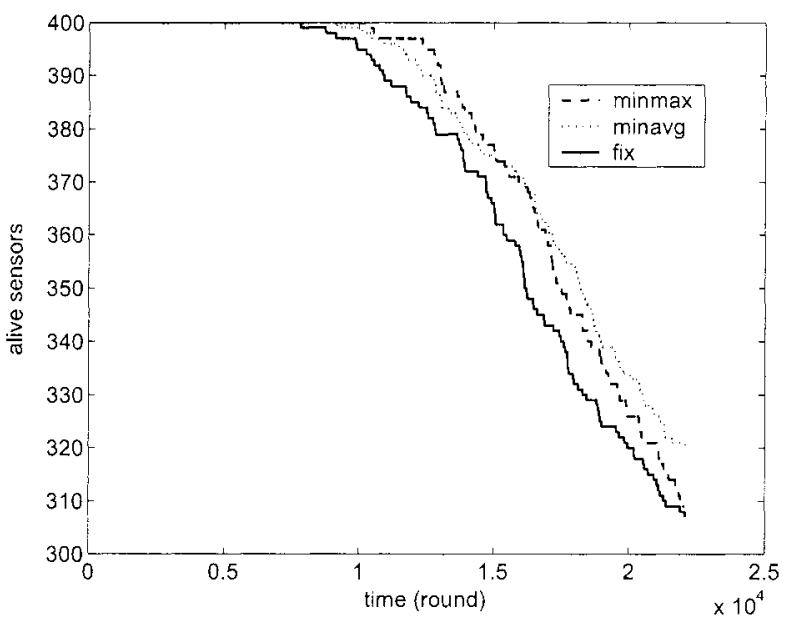

Figure 3. The alive sensors in the network

active in one round on the average, while in the second case that number is 20 nodes.

As shown in Figure 1, if the number of active nodes is low, both mobile BS strategies are significantly more energy-saving than the fixed BS solution. If the ratio of the active sensors is higher, then there is no major difference regarding the energy consumption.

Figure 2 shows the average energy consumption of one active sensor in one round, as a function of $p$.

When $p$ is low, the number of active sensors is also low. Therefore the two mobile BS strategies are significantly better than the fixed solution. For the individual sensors, both the minmax and the minavg strategies result in the energy consumption being decreased; therefore, the network lifetime is extended.

Figure 3 compares the number of alive sensors as a function of time in the case of the three strategies. The results show, that the first node dies first in the case of the fixed BS, at last in the case of minmax strategy. On the other hand with the minmax strategy the total energy of the network decreases more rapidly than with minavg; therefore, on the long run the minavg strategy proves to be a better choice. Depending on our goals, there are two possibilities to choose from. Minmax can be used if the main goal is to have the first node die at the latest possible moment in time. Meanwhile minavg can be used when we want to maximize the lifetime of the majority of the nodes.

We examined the performance of the strategies based on the time while the network operates as a function of the event intensity $(p)$ for different network 
lifetime definitions. Figure 4 shows the results of these simulations. We can see that, as the number of simultaneous events decreases (fewer active nodes), the minmax and minavg strategies outperform the fix placed BS more and more. This can be explained by the fact that if the number of active sensors is high, then it is more probable that there is an active sensor in every part of the cluster; hence, the optimal BS location is near the center of the network in case of both BS moving strategies. In contrast with that, having fewer active sensors results in a higher probability of having active sensors only in a smaller part of the cluster; thus, the optimal BS location is not in the center of the network.

The time elapsed till the depletion of the first node in the network is shown by Figure 4(a). It can be seen that the minmax strategy outperforms the minavg strategy for every value of $p$, except the lowest one. This is because, as mentioned earlier, the minmax strategy minimizes the maximum energy spent by a sensor in every round; this leads to a more balanced energy usage of the sensors, compared to the two other strategies. At the lowest event intensity the performance of the two mobile strategies is similar. The reason is that at this intensity the number of simultaneous events is about 2 ; therefore in most of the cases the optimal place is the same for both strategies. The results also show that the usage of the mobile BS prolongs the network lifetime more than $100 \%$ if the number of simultaneous events is low. Figure 4(b) shows the lifetime of the network for all the three strategies, supposing that the network is operable till $90 \%$ of the sensors are still alive. In this case both mobile strategies have approximately the same performance. However, if the lifetime of the network is defined as the time till $20 \%$ of the nodes die (Figure 4(c)), then the minavg strategy outperforms the other solutions. This is because the minavg strategy is places the BS always to the spot where the total energy required for the communication of the active sensors in the network is minimal, without taking into account the interests of individual sensors, as in minmax. Therefore, it can happen that some of them uses much more energy than the others, hence, some sensors may die sooner, while at the same time the majority of the sensors live longer compared to the minmax strategy. This operational difference leads to the effect that, as the operation of the network can tolerate more and more depleted sensors, the minavg strategy outperforms the minmax solution even more. Figure 4(d) shows the results that prove this statement.

In the next step of the performance evaluation we examined the effect of the two strategies on the distribution of the BS placement in the area of the cluster. We used long ran simulations, where the sensors had infinite energy; therefore, the depletion of the nodes did not bias the results. Figure 5 shows the distributions in the case of an event intensity of 0.0025 . The results show that the BS moves more often to the center of the cluster when the minmax strategy is used, while in the minavg strategy the of the BS is more evenly distributed in the cluster. It can be also seen that the peak of the distribution is in the center 


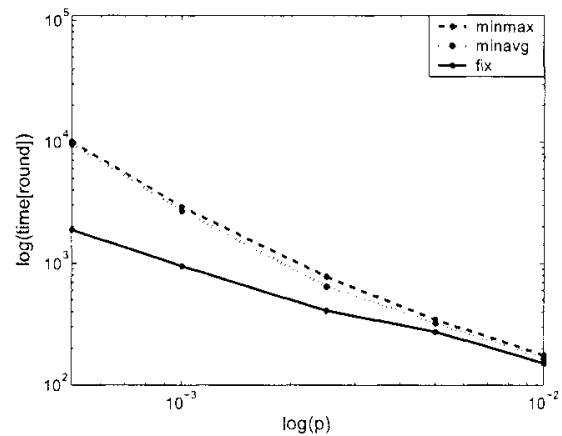

(a) first node dies

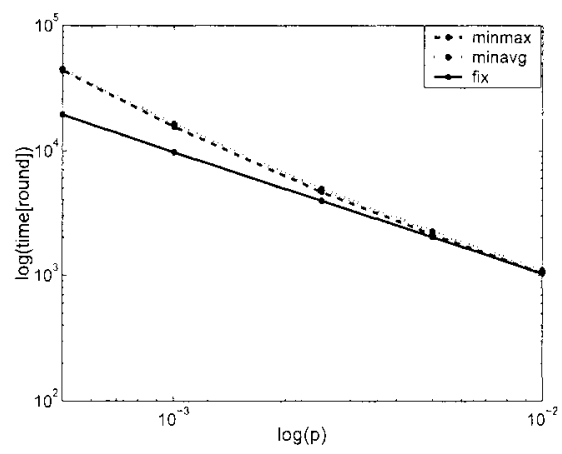

(c) $\gamma=20$

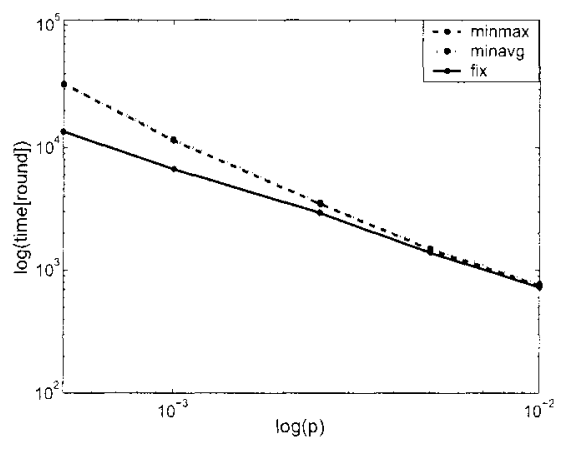

(b) $\gamma=10$

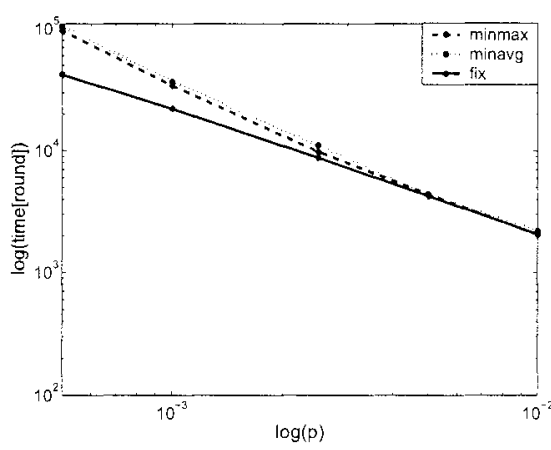

(d) $y=50$

Figure 4. Time till the $\gamma$ percent of sensors die, in function of the event intensity $(p)$

of the field in both cases. This is because the events appear independently in the area of the cluster; thus, it often happens that there is an active sensor in every part of the network.

For the explanation of the more balanced BS placement of the minavg strategy imagine the following. There are some active sensors close to each other in one part of the network. Meanwhile, there is one active sensor approximately equally far from the center, but on the opposite side of the network. If the optimal place of the BS is determined by the minavg strategy, then it is near to the group of the active sensors. If using the minmax strategy, the BS will be moved into the center area, minimizing the maximum distance.

Further results (not presented here), also suggest that decreasing the number of simultaneous events leads to a more balanced BS placement in the cluster. 


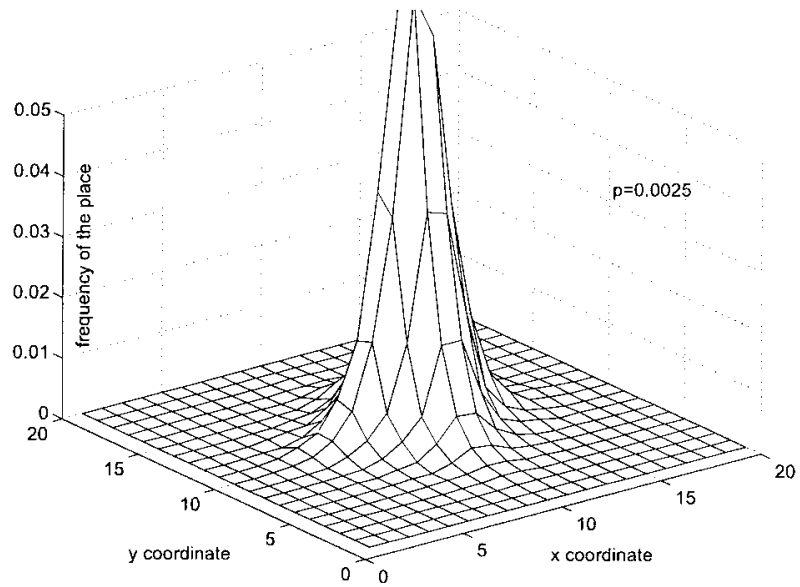

(a) minmax strategy

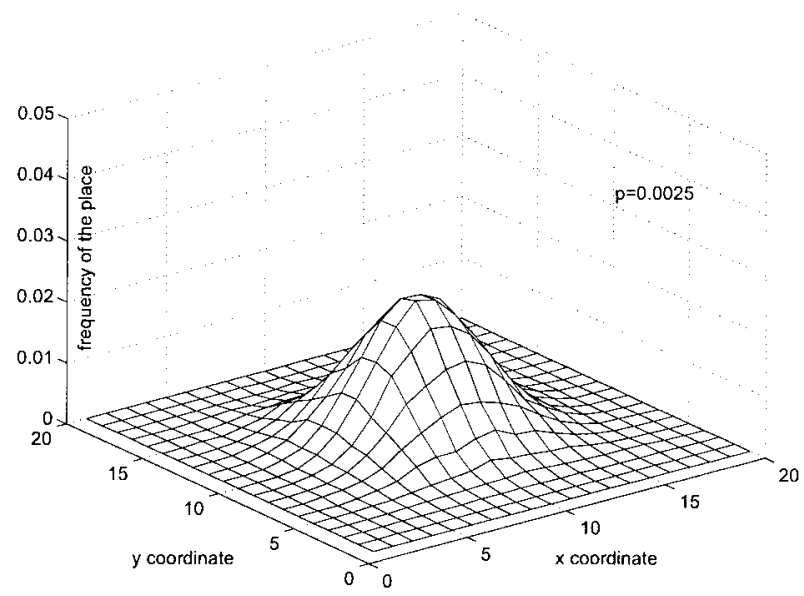

(b) minavg strategy

Figure 5. The distribution of the place of the BS 


\section{Conclusions and Future Work}

In this paper we presented the idea of moving the BS of a sensor network, in order to decrease the amount of energy required for communication, and hence prolong the lifetime of the network. We introduced two different strategies for moving the BS: minmax and minavg. The first one minimizes the maximum energy consumption of one active sensor, while the other one minimizes the average energy required for the communication between the BS and the active sensors.

After the introduction of the strategies we presented simulation results evaluating their performance. The results have shown that if the number of simultaneous events is low, then the minmax and the minavg strategies outperform the fix placed BS and significantly prolong the lifetime of the network. From the results it can be established also that the minmax strategy has the better performance if the network is operable only while every sensor is alive. On the contrary, the minavg strategy performs better if the operation of the network is able to tolerate the depletion of some sensors.

In this work we considered only direct communication in the network. One of our future goals is to examine the applicability and the performance of the two mobile BS strategies in sensor networks where multihop communication is also present. As future work other BS moving strategies will be examined, which also consider the depletion of sensors. Another possible future direction is to examine the case when the event in the sensor network is sensed not only by one sensor, but by a larger number of neighboring sensors.

\section{References}

Al-Karaki, J. and Kamal, A. (2004). A Taxonomy of Routing Techniques in Wireless Sensor Networks. Sensor Networks Handbook. CRC Publishers.

Carson, Y. and Maria, A. (1997). Simulation optimization: Methods and applications. In Proc. 9 th conference on Winter Simulation, pages 118-126, Atlanta, USA.

Doherty, L., Warneke, B. A., Boser, B. E., and Pister, K. (2001). Energy and performance considerations for smart dust. International Journal of parallel Distributed Systems and Networks, 4(3):121-133.

Gan, L., Liu, J., and Jin, X. (2004). Agent-based, energy efficient routing in sensor networks. In Proc. 3rd International Joint Conference on Autonomous Agents and Multiagent Systems (AAMAS'04), volume 1, pages 472-479, New York, USA.

Gandham, S., Dawande, M., and Prakash, R. (2004). An integral flow-based energy-efficient routing algorithm for wireless sensor networks. In Proc. IEEE Wireless Communications and Networking Conference (WCNC'04), volume 5, pages 2344-2349, Atlanta, USA.

Ganesan, D., Govindan, R., Shenker, S., and Estrin, D. (2001). Highly-resilient, energy-efficient multipath routing in wireless sensor networks. ACM SIGMOBILE Mobile Computing and Communications Review, 5(4):11-25. 
Howard, A., Mataric, M., and Sukhatme, G. (2002). Mobile sensor network deployment using potential fields: A distributed, scalable solution to the area coverage problem. In Proc. Distributed Autonomous Robotic Systems (DARS'02), pages 299-308, Fukuoka, Japan.

Intanagonwiwat, C., Govindan, R., and Estrin, D. (2000). A scalable and robust communication paradigm for sensor networks. In Proc. 6th Annual International Conference on Mobile Computing and Networks (MobiCOM 2000), pages 56-67, Boston, USA.

Kim, Hyung Seok, Abdelzaher, Tarek F., and Kwon, Wook Hyun (2003). Minimum-energy asynchronous dissemination to mobile sinks in wireless sensor networks. In SenSys '03. Proceedings of the Ist international conference on Embedded networked sensor systems, pages 193-204, New York, USA.

Megiddo, N. (1983). The weighted euclidean 1-center problem. Mathematics of Operations Research, 8(4):498-504.

Rahimi, M., Shah, H., Sukhatme, G., Heidemann, J., and Estrin, D. (2003). Studying the feasibility of energy harvesting in a mobile sensor network. In Proc. IEEE International Conference on Robotics and Automation, pages 19-24, Taipai.

Rahul, C. and Rabaey, J. (2002). Energy aware routing for low energy ad hoc sensor networks. In Proc. IEEE Wireless Communications and Networking Conference (WCNC'02), volume 1, pages $350-355$, Orlando, USA.

Rao, S., Gandham, S., Dawande, M., Prakash, R., and Venkatesan, S. (2003). Energy efficient schemes for wireless sensor networks with multiple mobile base stations. In Proc. IEEE Globecom, volume 22, pages 377-381, San Francisco, USA.

Schurgers, C. and Srivastava, M. B. (2001). Energy efficient routing in wireless sensor networks. In MILCOM Proc. on Communications for Network-Centric Operations: Creating the Information Force, pages 28-31, McLean.

Wang, Z. Maria, Basagni, Stefano, Melachrinoudis, Emanuel, and Petrioli, Chiara (2005). Exploiting sink mobility for maximizing sensor networks lifetime. In HICSS '05: Proceedings of the Proceedings of the 38th Annual Hawaii International Conference on System Sciences (HICSS'05) - Track 9, page 287.1, Washington, USA.

Yao, Y. and Gehrke, J. (2002). The cougar approach to in-network query processing in sensor networks. SIGMOD Record, 31(3):9-18.

Ye, Fan, Luo, Haiyun, Cheng, Jerry, Lu, Songwu, and Zhang, Lixia (2002). A two-tier data dissemination model for large-scale wireless sensor networks. In MobiCom '02: Proceedings of the 8th annual international conference on Mobile computing and networking, pages 148159, New York, USA.

Zhao, F. and Gubias, L. (2004). Wireless Sensor Networks: An Information Processing Approach. Elsevier. 